\title{
Why Are There People With Psychosis And People Without? Is It All Due To Genetic And Vulnerability Factors?
}

\author{
Edo S. Jaya \\ Psychology Research Method Department, Faculty of Psychology, Universitas Indonesia, \\ Depok, Indonesia
}

*Corresponding Author:

Edo S. Jaya

Psychology Research Method Department

Faculty of Psychology, Universitas Indonesia

Jl. Lkr. Kampus Raya, Depok, Jawa Barat

Indonesia, 16424

Tel.: +62 217270004

Email address: edo.jaya@ui.ac.id 


\title{
Why are there people with psychosis and people without? Is it all due to genetic and vulnerability factors?
}

\begin{abstract}
Schizophrenia or psychotic disorder has often been assumed as a hereditary disorder. However, recently accumulated evidence has demonstrated the importance of environmental factors, particularly social factors. Two dominant theoretical frameworks explain the association between social factors and psychosis, i.e., the social defeat (SD) hypothesis and cognitive models of psychosis. According to SD hypothesis, the common denominator of most social risk factors is the negative experience of being excluded from a majority group. According to cognitive models, psychosis occurs due to non-adaptive appraisals of anomaly experience. Recent studies suggest that these explanations complement each other. However, application of these frameworks in explaining cases in clinical setting has not been examined. In this single-case research design study, a participant diagnosed with schizophrenia from Bogor, Indonesia was interviewed for his medical history (anamneses). The psychological model of SD was used to comprehend anamneses. The root of psychotic symptoms of the participant was familial conflict, which led to his negative beliefs about himself and others. This negative view of himself and others caused the patient to experience clinical level of negative affect, which in turn developed into psychotic symptoms. To conclude, the psychological model of SD can be used to assess clients in the clinic.
\end{abstract}

Keywords: schizophrenia; cognitive behavioral therapy; CBT; childhood trauma; mechanism; dopamine

\section{Introduction}

According to the Diagnostic and Statistical Manual of Mental Disorders 5, schizophrenia can be diagnosed if an individual presents notably himself with at least two of the following symptoms in a one-month period: delusion, hallucination, disorganized speech, grossly disorganized or catatonic behavior, and negative symptoms (American Psychiatric Association, 2013). At least one of these symptoms must be delusions, hallucinations, or disorganized speech. However, the symptoms that are most significant in clinical practice arguably include persecutory delusion (Freeman \& Garety, 2014) and hallucinations (Beck \& Rector, 2003). Persecutory delusion has been reported to be experienced by $70 \%$ of first-episode psychosis patients (Coid et al., 2013), and $70 \%$ of schizophrenia patients reported to have experienced hallucinations (Sartorius et al., 1986).

Although having such diagnostic category aids the healthcare system and clinical practice in a helicopter perspective, categorizing individuals with and without schizophrenia is not consistently helpful in research and individual clinical experience. The view that schizophrenia can only occur in either-or state has been contested for some time. For example, the idea that delusions and hallucinations are discrete, discontinuous phenomena with nothing in between has been challenged since 50 years ago (Strauss, 1969). Most recently, this work has been continued and improved into a theory of a continuum of psychosis, particularly according to the work of van Os and colleagues (Johns \& van Os, 2001; Linscott \& van Os, 2010). 
Multiple theories explain the continuum of psychosis, and this notion features several important tenets. First, the idea of a psychosis continuum does not mean a continuum of disorder. Although $5.8 \%$ of the general population in 18 countries reports some kind of psychotic experiences (McGrath et al., 2015), only a very small fraction $(0.55 \%)$ is diagnosed with schizophrenia (McGrath, Saha, Chant, \& Welham, 2008). Second, the psychosis continuum does not follow a Gaussian distribution. Specifically, the psychosis continuum should be positively skewed (Johns \& van Os, 2001). This condition implies that most people should have no-to-very-low level of symptoms, but a significant proportion of the population yields non-zero values. Importantly, this hypothesized distribution has been demonstrated exactly as predicted with regard to paranoid thoughts in the general population (Freeman et al., 2005). Third, at the subclinical level, psychotic symptoms, also called psychotic experiences, are argued to be on the same continuum with psychotic disorder. Psychotic symptoms experienced by individuals without diagnosis of psychotic disorder differ only in frequency, severity, and/or distress in comparison with the symptoms experienced by those with diagnosis. In other words, these individuals show no difference in kind and cause of psychotic symptoms. For example, a meta-analysis using odds ratios from various studies investigating risk factors of psychotic symptoms showed that such risk factors exhibit high similarity to those of schizophrenia (Linscott \& van Os, 2013). This similarity of etiology assumption is strengthened with evidence from a twin study showing that both severe and non-severe psychotic experiences are hereditary (Zavos et al., 2014).

Despite years of research, the question on determining the causes of psychotic disorder has not arrived at a conclusive answer. Drawing from classic twin studies, psychotic disorder has been suggested to be highly heritable (Tandon, Keshavan, \& Nasrallah, 2008); however, recent findings from genome-wide association studies suggest that the results may have been overestimated (Schizophrenia Working Group of the Psychiatric Genomics Consortium, 2014). More recently, accumulated evidence has suggested the importance of environmental factors, particularly social factors.

One main reason for the creation of vulnerability-stress paradigm (Zubin, 1986) was to integrate heterogeneous explanations for clinical disorders. At the time of development, seven types of etiological explanations for clinical disorders were available, spanning from the molecular biological pole to the environment pole, which comprise the genetic model, internal environment (infectious disease or virus), neurophysiological, learning theory, developmental, and sociocultural model. At present, the vulnerability-stress paradigm is still used to guide research and explain the related findings. For example, Clamor and colleagues (Clamor et al., 2014; Clamor, Lincoln, Thayer, \& Koenig, 2016) used the vulnerability-stress paradigm to explain the findings that low heart-rate variability is a unique psychosis symptom. Specifically, heart-rate variability can be considered both as a vulnerability marker and stress response that occurs prior to psychotic symptoms.

Although the vulnerability-stress paradigm has guided research and motivated researchers to integrate research findings from various disciplines, it fails to specify the types of vulnerability and stress. The current trend of adopting all factors as potential causes of clinical disorder (given that most factors can be either placed under the category of vulnerability or stress) has been criticized as not helpful in advancing our knowledge of clinical disorders (see Kendler, 2012). Therefore, several reasons are believed when considering the possible usefulness of adopting a new paradigm. 
The social defeat hypothesis (SD, Selten, van der Ven, Rutten, \& Cantor-Graae, 2013) is a theoretical framework that attempts to explain findings from various studies revealing the different environmental factors of psychosis; these factors include migration (Cantor-Graae \& Selten, 2005), childhood trauma (Arseneault et al., 2011; Lincoln, Marin, \& Jaya, 2017), and cannabis use (Moore et al., 2007). The SD hypothesis states that the common denominator of these risk factors is the negative experience of being excluded from a majority group. This notion can be traced to the well-known phenomenon from animal experimental studies, which showed that rodents exposed to stress induced with SD (e.g., forcing them to live with a dominant rodent) exhibited behavioral markers of psychosis, such as fear of other rodents, similar to paranoia (Badowska, Brzózka, Chowdhury, Malzahn, \& Rossner, 2015).

Although some arguments in the theoretical framework of SD hypothesis state that SD is causally related to psychosis, establishing causality is particularly difficult in clinical psychology and psychiatry. To establish the causal relation of SD to psychosis, as stated by Selten et al. (2013), two alternative explanations to the association between SD risk factors and psychosis must be examined: 1) the observed association results from genetic confounding, and 2) SD is not a specific risk factor for psychosis. In other words, SD is a consequence rather than a cause of psychosis. First, the genetic confounding explanation assumes that individuals who are genetically predisposed to psychosis move to urban areas, emigrate due to failure to integrate in home country (i.e., social selection hypothesis (Ødegård, 1932), are victimized during childhood due to poor social skills, and use illicit drugs because of unhappiness. However, this explanation requires the discovery of a group of genes that is responsible for many factors and at the same time, cause schizophrenia, transfer to urban areas, emigration, acts of bullying, and drug use; such group of genes is unlikely to be discovered. Second, risk factors are rarely specific in clinical psychology and psychiatry. Non-specific risk factors also exist in the field of medicine, for example, smoking has been found to cause various forms of cancer, cardiovascular diseases, and respiratory diseases (Office of the Surgeon General (US) \& Office on Smoking and Health (US), 2004); thus, smoking is a non-specific but causal risk factor.

Pioneering psychological models explaining the development of various psychosis symptoms have been proposed decades ago (e.g., Mintz \& Alpert, 1972). Since then, research findings on psychological factors of psychosis have accumulated and led to advancements and better understanding of psychological models. One group of psychological models, called cognitive models of psychosis, have been proven useful and became a basis of psychological therapy for psychosis, called cognitive behavioral therapy for psychosis (CBTp), which has become widely recommended in the UK (National Institute for Health and Clinical Excellence, 2009). Many cognitive models of psychosis have been developed, and each focuses on certain aspect of the symptoms, for example, hallucination (Beck \& Rector, 2003), persecutory delusion (Kesting \& Lincoln, 2013), and positive symptoms (Garety, Bebbington, Fowler, Freeman, \& Kuipers, 2007).

However, none of these models has focused on social risk factors of psychosis and its mechanism. In my dissertation, I attempted to formulate the psychological mechanism of the SD hypothesis (see Jaya, 2017). The model can be found in my dissertation. Briefly, the model starts on the left-hand side with an ellipse (SD) that comprises different social adversities. This section of the model reflects the findings from confirmatory factor analyses, which showed that SD is a common denominator of various social adversities (for details, see Jaya \& Lincoln, 2016). Then, 
an arrow spans from SD to the negative schema and psychotic symptoms. Specifically, the association between SD and psychotic symptoms is partially mediated by negative schemas (as shown in Jaya, Ascone, \& Lincoln, 2017b). Finally, negative schemas are hypothesized to exert an indirect influence via negative affect toward psychotic symptoms (as shown in Jaya, Ascone, $\&$ Lincoln, 2017a). Negative affect plays both the role of a mediator, which translates the effect of negative schemas to psychotic symptoms, and a maintenance factor that forms a vicious cycle of negative schemas and negative affect reinforcing each other over time. In short, social adversities can influence psychotic symptoms via SD experience, and a vicious cycle of negative schemas and negative affect explains this relationship.

The therapeutic implication of this model is to focus on targeting negative schemas and negative affect while acknowledging the important role of social adversities as precipitants of psychotic symptoms. This condition supports the current practice of CBTp, which is manualized and based on separate techniques. For example, negative schemas can be targeted with techniques, such as cognitive restructuring, and negative affect can be targeted with techniques, such as emotional regulation techniques. However, a central element of CBTp approach is the importance of case formulation before therapy. In the next section, I demonstrate the feasibility of the psychological model of SD in understanding clients in the clinic.

\section{Methods}

\section{Participant}

The participant was a male farmer from the outer Bogor region in Indonesia. He was 35 years old and with elementary school education. He was married twice, and both marriages ended in divorce; each marriage bore one child who lived with their mothers. Initially, the patient was diagnosed with hebephrenic schizophrenia (i.e., disorganized schizophrenia, DSM-IV-TR). However, his disorganized behavioral symptoms improved quickly, and his diagnosis at the time was changed to paranoid schizophrenia. The patient received regular medications consisting of typical and atypical antipsychotics, a standard treatment in the hospital that he attended.

The participant reported no family history of psychosis. He said that none of his family members (including extended family) had been admitted to a mental hospital. This claim was supported by the data gathered from an interview with his sister, who was his closest family member. The sister also claimed that no family member had shown symptoms similar to those of the patient's. Similarly, the participant's grandmother confirmed that she knew no one from the family who experiences symptoms similarly to the patient.

\section{Research Design}

The research design was a single-case clinical study. The study aimed to understand the medical history (anamneses) of the participant using the psychological model of SD hypothesis.

\section{Measures}

Clinical interview and observation were used to gather data. 


\section{Procedure}

The participant was observed and interviewed in a mental hospital in Bogor region in Indonesia. The participant's main caregiver (his sister) and grandmother were also interviewed to gather further information about the participant and to confirm the information provided by the participant.

\section{Data Analysis}

Medical history data were analyzed qualitatively.

\section{Results}

Using the psychological model of the SD hypothesis, the medical history of the participant was formulated, as shown in Figure 1. The origin of the psychotic symptoms of the patient was traced back to his conflict within his family, from which he experienced severe social undermining and ostracism. These experiences were appraised non-adaptively and triggered the development of negative schemas. The negative schemas in turn fed into the vicious cycle of negative schemas and negative affect, which finally led to the development of psychotic symptoms, such as paranoid thinking, grandiosity, magical thinking, and hallucination.

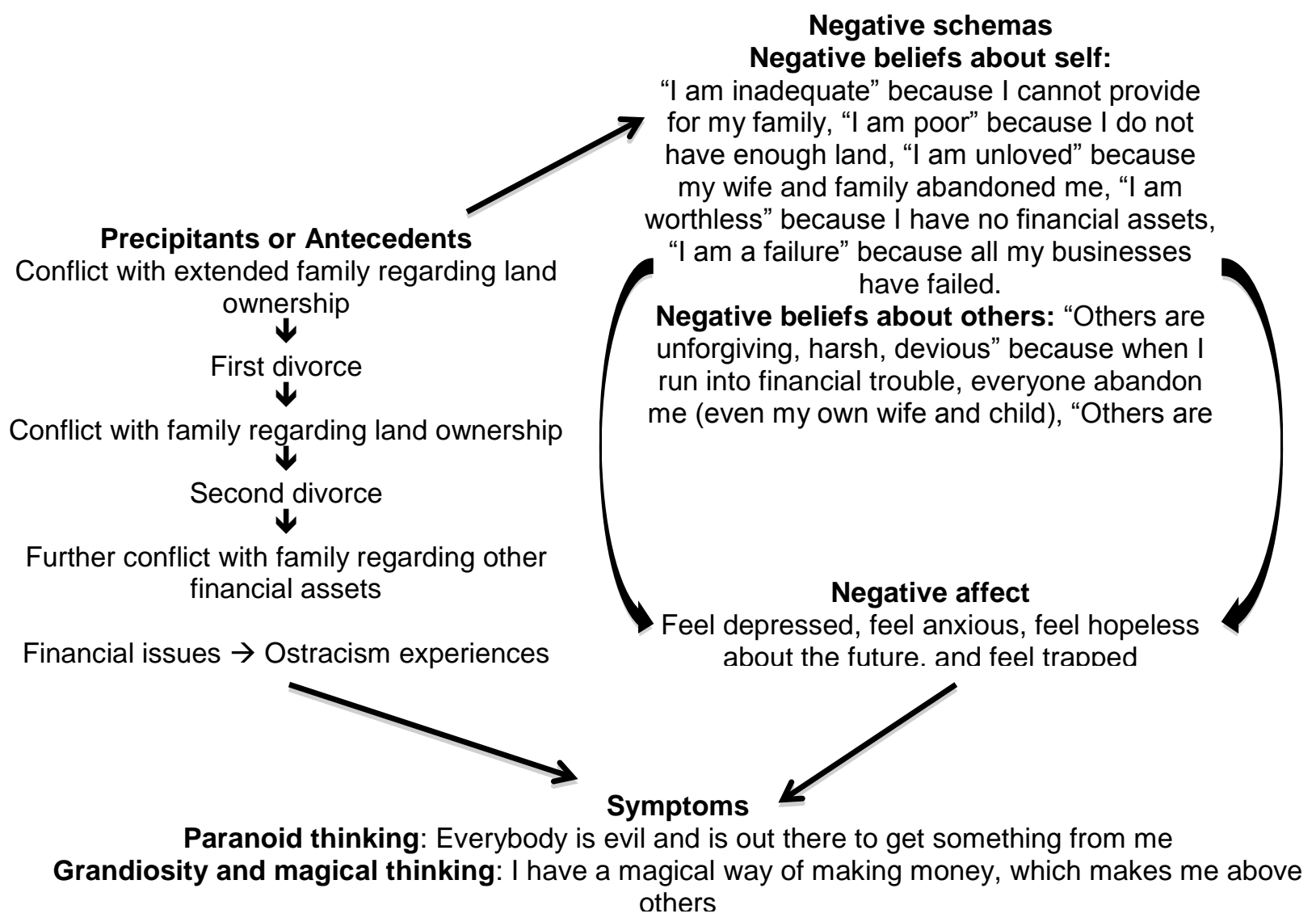

Figure 1. Case formulation of the participant's symptom development 


\section{Discussion}

To conclude, psychotic disorders may not be a primarily genetic disorder and that a psychological explanation can be both valid and useful in clinical practice. In this paper, the outlined psychological mechanism of social defeat can be used to explain the development and possible maintenance of psychotic symptoms. Therapies that specifically target negative beliefs about self and others could be particularly effective in managing psychotic symptoms; such therapies include cognitive restructuring techniques from cognitive behavioral therapy regiments. Techniques such as emotional regulation techniques, mood monitoring, and relaxation techniques could also be effective in halting the effect of social defeat and negative schemas on psychotic symptoms. Until the time of writing, only one published trial has targeted negative schemas or negative affect for treatment of psychosis (i.e., Freeman et al., 2014), but it focused on paranoia and was based on low sample number. Future studies should conduct trials with stronger power.

\section{References}

American Psychiatric Association. (2013). Diagnostic and Statistical Manual of Mental Disorders (DSM-5®). American Psychiatric Pub.

Arseneault, L., Cannon, M., Fisher, H. L., Polanczyk, G., Moffitt, T. E., \& Caspi, A. (2011). Childhood trauma and children's emerging psychotic symptoms: A genetically sensitive longitudinal cohort study. American Journal of Psychiatry, 168(1), 65-72. https://doi.org/10.1176/appi.ajp.2010.10040567

Badowska, D. M., Brzózka, M. M., Chowdhury, A., Malzahn, D., \& Rossner, M. J. (2015). Data calibration and reduction allows to visualize behavioural profiles of psychosocial influences in mice towards clinical domains. European Archives of Psychiatry and Clinical Neuroscience, 265(6), 483-496. https://doi.org/10.1007/s00406-014-0532-6

Beck, A. T., \& Rector, N. A. (2003). A cognitive model of hallucinations. Cognitive Therapy and Research, 27(1), 19-52. https://doi.org/10.1023/A:1022534613005

Cantor-Graae, E., \& Selten, J.-P. (2005). Schizophrenia and migration: A meta-analysis and review. American Journal of Psychiatry, 162(1), 12-24. https://doi.org/10.1176/appi.ajp.162.1.12

Clamor, A., Hartmann, M. M., Köther, U., Otte, C., Moritz, S., \& Lincoln, T. M. (2014). Altered autonomic arousal in psychosis: An analysis of vulnerability and specificity. Schizophrenia Research, 154(1-3), $73-78$. https://doi.org/10.1016/j.schres.2014.02.006

Clamor, A., Lincoln, T. M., Thayer, J. F., \& Koenig, J. (2016). Resting vagal activity in schizophrenia: meta-analysis of heart rate variability as a potential endophenotype. The British Journal of Psychiatry, 208(1), 9-16. https://doi.org/10.1192/bjp.bp.114.160762

Coid, J. W., Ullrich, S., Kallis, C., Keers, R., Barker, D., Cowden, F., \& Stamps, R. (2013). The relationship between delusions and violence: Findings from the East London first episode psychosis study. JAMA Psychiatry, 70(5), $465-471$. https://doi.org/10.1001/jamapsychiatry.2013.12

Freeman, D., \& Garety, P. A. (2014). Advances in understanding and treating persecutory delusions: A review. Social Psychiatry and Psychiatric Epidemiology, 49(8), 1179-1189. https://doi.org/10.1007/s00127-014-0928-7

Freeman, D., Garety, P. A., Bebbington, P. E., Smith, B., Rollinson, R., Fowler, D., ... Dunn, G. (2005). Psychological investigation of the structure of paranoia in a non-clinical population. British Journal of Psychiatry, 186(5), 427-435.

Freeman, D., Pugh, K., Dunn, G., Evans, N., Sheaves, B., Waite, F., ... Fowler, D. (2014). An early Phase II randomised controlled trial testing the effect on persecutory delusions of using CBT to reduce negative cognitions about the self: The potential benefits of enhancing self confidence. Schizophrenia Research, 160(1-3), 186-192. https://doi.org/10.1016/j.schres.2014.10.038

Garety, P. A., Bebbington, P., Fowler, D., Freeman, D., \& Kuipers, E. (2007). Implications for neurobiological research of cognitive models of psychosis: a theoretical paper. Psychological Medicine, 37(10), $1377-1391$. https://doi.org/10.1017/S003329170700013X

Jaya, E. S. (2017, July 6). The interplay between social factors and psychotic symptoms: Cognitive vulnerability and affective pathway in focus (Unpublished Dissertation). Universität Hamburg, Hamburg, Germany. Retrieved from http://ediss.sub.uni-hamburg.de/volltexte/2017/8581/

Jaya, E. S., Ascone, L., \& Lincoln, T. M. (2017a). A longitudinal mediation analysis of the effect of negative-self-schemas on positive symptoms via negative affect. Psychological Medicine, 1-11. https://doi.org/10.1017/S003329171700277X

Jaya, E. S., Ascone, L., \& Lincoln, T. M. (2017b). Social adversity and psychosis: The mediating role of cognitive vulnerability. Schizophrenia Bulletin, 43(3), 557-565. https://doi.org/10.1093/schbul/sbw104 
Jaya, E. S., \& Lincoln, T. M. (2016). Social adversities and psychotic symptoms: A test of predictions derived from the social defeat hypothesis. Psychiatry Research, 245, 466-472. https://doi.org/10.1016/j.psychres.2016.09.002

Johns, L. C., \& van Os, J. (2001). The continuity of psychotic experiences in the general population. Clinical Psychology Review, 21(8), 1125-1141. https://doi.org/10.1016/S0272-7358(01)00103-9

Kendler, K. S. (2012). The dappled nature of causes of psychiatric illness: replacing the organic-functional/hardware-software dichotomy with empirically based pluralism. Molecular Psychiatry, 17(4), $377-388$. https://doi.org/10.1038/mp.2011.182

Kesting, M.-L., \& Lincoln, T. M. (2013). The relevance of self-esteem and self-schemas to persecutory delusions: A systematic review. Comprehensive Psychiatry, 54(7), 766-789. https://doi.org/10.1016/j.comppsych.2013.03.002

Lincoln, T. M., Marin, N., \& Jaya, E. S. (2017). Childhood trauma and psychotic experiences in a general population sample: A prospective study on the mediating role of emotion regulation. European Psychiatry, 42, 111-119. https://doi.org/10.1016/j.eurpsy.2016.12.010

Linscott, R. J., \& van Os, J. (2010). Systematic reviews of categorical versus continuum models in psychosis: evidence for discontinuous subpopulations underlying a psychometric continuum. Implications for DSM-V, DSM-VI, and DSMVII. Annual Review of Clinical Psychology, 6(1), 391-419. https://doi.org/10.1146/annurev.clinpsy.032408.153506

Linscott, R. J., \& van Os, J. (2013). An updated and conservative systematic review and meta-analysis of epidemiological evidence on psychotic experiences in children and adults: On the pathway from proneness to persistence to dimensional expression across mental disorders. Psychological Medicine, 43(6), 1133-1149. https://doi.org/10.1017/S0033291712001626

McGrath, J. J., Saha, S., Al-Hamzawi, A., Alonso, J., Bromet, E. J., Bruffaerts, R., ... Kessler, R. C. (2015). Psychotic experiences in the general population: A cross-national analysis based on 31261 respondents from 18 countries. JAMA Psychiatry, 72(7), 697-705. https://doi.org/10.1001/jamapsychiatry.2015.0575

McGrath, J. J., Saha, S., Chant, D., \& Welham, J. (2008). Schizophrenia: a concise overview of incidence, prevalence, and mortality. Epidemiologic Reviews, 30(1), 67-76. https://doi.org/10.1093/epirev/mxn001

Mintz, S., \& Alpert, M. (1972). Imagery vividness, reality testing, and schizophrenic hallucinations. Journal of Abnormal Psychology, 79(3), 310-316.

Moore, T. H., Zammit, S., Lingford-Hughes, A., Barnes, T. R., Jones, P. B., Burke, M., \& Lewis, G. (2007). Cannabis use and risk of psychotic or affective mental health outcomes: A systematic review. The Lancet, 370(9584), 319-328. https://doi.org/10.1016/S0140-6736(07)61162-3

National Institute for Health and Clinical Excellence. (2009). Schizophrenia: Core Interventions in the Treatment and Management of Schizophrenia in Primary and Secondary Care (Update). Leicester (UK): British Psychological Society. Retrieved from http://www.ncbi.nlm.nih.gov/books/NBK11681/

Ødegård, Ø. (1932). Emigration and insanity: a study of mental disease among the Norwegian born population of Minnesota. Copenhagen: Levin \& Munksgaard.

Office of the Surgeon General (US), \& Office on Smoking and Health (US). (2004). The Health Consequences of Smoking: A Report of the Surgeon General. Atlanta (GA): Centers for Disease Control and Prevention (US). Retrieved from http://www.ncbi.nlm.nih.gov/books/NBK44695/

Sartorius, N., Jablensky, A., Korten, A., Ernberg, G., Anker, M., Cooper, J. E., \& Day, R. (1986). Early manifestations and firstcontact incidence of schizophrenia in different cultures. A preliminary report on the initial evaluation phase of the WHO Collaborative Study on determinants of outcome of severe mental disorders. Psychological Medicine, 16(4), 909-928.

Schizophrenia Working Group of the Psychiatric Genomics Consortium. (2014). Biological insights from 108 schizophreniaassociated genetic loci. Nature, 511(7510), 421-427. https://doi.org/10.1038/nature13595

Selten, J.-P., van der Ven, E., Rutten, B. P. F., \& Cantor-Graae, E. (2013). The social defeat hypothesis of schizophrenia: An update. Schizophrenia Bulletin, 39(6), 1180-1186. https://doi.org/10.1093/schbul/sbt134

Strauss, J. S. (1969). Hallucinations and delusions as points on continua function: Rating scale evidence. Archives of General Psychiatry, 21(5), 581-586. https://doi.org/10.1001/archpsyc.1969.01740230069010

Tandon, R., Keshavan, M. S., \& Nasrallah, H. A. (2008). Schizophrenia, "Just the Facts" What we know in 2008.2. Epidemiology and etiology. Schizophrenia Research, 102(1-3), 1-18. https://doi.org/10.1016/j.schres.2008.04.011

Zavos, H. M. S., Freeman, D., Haworth, C. M. A., McGuire, P., Plomin, R., Cardno, A. G., \& Ronald, A. (2014). Consistent etiology of severe, frequent psychotic experiences and milder, less frequent manifestations: a twin study of specific $\begin{array}{lllll}\text { psychotic } & \text { experiences in } 1049-1057 .\end{array}$ https://doi.org/10.1001/jamapsychiatry.2014.994

Zubin, J. (1986). Models for the etiology of schizophrenia. In G. D. Burrows, T. R. Norman, \& G. Rubinstein (Eds.), Handbook of Studies on Schizophrenia: Epidemiology, aetiology, and clinical features (pp. 97-104). Oxford: Elsevier. 